TiC-MgO composite: An X-ray transparent and machinable heating element in a 7 France

$8 \quad{ }^{2}$ Institute for Planetary Materials, Okayama University, Misasa, Tottori 682-0193, Japan 11 France

\section{multi-anvil high pressure apparatus}

Fang $\mathrm{Xu}^{1}$, Longjian $\mathrm{Xie}^{2 *}$, Akira Yoneda ${ }^{2}$, Nicolas Guignot ${ }^{3}$, Andrew King ${ }^{3}$, Guillaume Morard $^{1 \S}$, Daniele Antonangeli ${ }^{1}$

${ }^{1}$ Sorbonne Université, Muséum National d'Histoire Naturelle, UMR CNRS 7590, Institut de Minéralogie, de Physique des Matériaux et de Cosmochimie, IMPMC, 75005 Paris, * Currently at Bayerisches Geoinstitut, Universität Bayreuth, 95440 Bayreuth, Germany

${ }^{3}$ Synchrotron SOLEIL, L'Orme de Merisiers, Saint Aubin-BP48, 91192 Gif-sur-Yvette,

$\S$ Currently at Institut des Sciences de la Terre (ISTerre), Université Grenoble-Alpes, 1381 rue de la scine F-38610 Gières

\section{Abstract}

TiC-MgO composite was developed as a heating element for X-ray study in the multianvil high pressure apparatus. We synthesized TiC-MgO blocks (50-70 wt.\% of TiC) by compression in a cold isostatic press followed by baking in a gas flow furnace. Heaters of tubular shape were manufactured from the synthesized blocks either by lathe or numerically controlled milling machine from synthesized blocks. The so-produced heating elements have been proved to generate temperatures up to $2250 \mathrm{~K}$ at $10 \mathrm{GPa}$, condition where classical graphite heaters are not suitable anymore due to graphite-diamond transition. These new 
23 heaters have been successfully used for in situ X-ray radiography and diffraction 24 measurements on liquid Fe alloys, exploiting excellent X-ray transparency.

25

26 Key words: multi-anvil; heater; TiC-MgO composite; X-ray transparency; high temperature 27 


\section{Introduction}

Pressure and temperature are fundamental thermodynamic variables that largely

30 affect elastic, electronic, magnetic, structural and chemical properties of materials.

31 Experiments under simultaneous high-pressure and high-temperature conditions are

32 necessary to complement geophysical observations to reveal structures and properties of the

33 Earth's deep interiors. Multi-anvil apparatus can compress large cell assembly with fine

34 temperature control and reduced thermal gradients compared with diamond anvil cell, and

35 over a significantly larger pressure and temperature range compared with Paris-Edinburgh

36 press [1]. Last decades witnessed substantial technical developments in experimentation in

37 multi-anvil apparatus, in particular in combination with the in situ X-ray observations,

38 showing great versatility, with studies ranging from phase diagram and equation of state by

39 X-ray diffraction (see review by [2]), to viscosity measurement by X-ray radiography [e.g. 3,

40 4], and density determination in liquids by X-ray absorption [e.g. 5, 6].

41 X-ray transparent heating elements are required to conduct in situ X-ray

42 measurements in the multi-anvil apparatus at high temperature. Graphite is the most

43 conventional heater for X-ray measurements under high pressures because of its high X-ray

44 transparency. However, graphite heaters suffer malfunction at $\sim 10 \mathrm{GPa}$ because of

45 transformation to diamond, which limits the use of such heaters over an extended pressure

46 range. Metallic (Mo, Re and Ta) and lanthanum chromite $\left(\mathrm{LaCrO}_{3}\right)$ heaters are widely used

47 as heating elements in high-pressure laboratory experiments. However, they are not suitable

48 for in situ X-ray observations because of their low X-ray transparency. Recently, Xie et al.

49 [7] developed boron-doped diamond (BDD) heater, which is very refractory and X-ray 
transparent. However, owing to its hardness, it is hard to machine, making the production of

51 tubular heaters out of $\mathrm{BDD}$ difficult. $\mathrm{TiB}_{2}+\mathrm{BN}$ composite (e.g. EBN grade from DENKA

52 Ltd.) is widely used as a commercially available X-ray transparent heating element in high

53 pressure experiments. However, the highest achievable temperature is limited to $\sim 2000 \mathrm{~K}$.

54 In summary, there is a clear need to develop an alternative heater for in situ X-ray

55 experiments at pressure and temperature exceeding $10 \mathrm{GPa}$ and $2000 \mathrm{~K}$.

Titanium carbide, TiC, is a highly refractory material having sodium chloride

57 structure at ambient conditions [8]. The high melting point (3100 K at ambient pressure) [9],

58 its low density $\left(4.93 \mathrm{~g} / \mathrm{cm}^{3}\right)$ and the absence of phase transformation up to $90 \mathrm{GPa}$ [10],

59 makes TiC a promising material for an X-ray transparent heating element. Taniguchi et al.

60 [11] developed a TiC-diamond heater and successfully generated $2273 \mathrm{~K}$ at $10 \mathrm{GPa}$ in a

61 large-volume belt-type high pressure apparatus. In this study, TiC-diamond composite was

62 synthesized by heat treatment of a mixture of diamond and non-stoichiometric $\mathrm{TiC}_{1-\mathrm{x}}(\mathrm{x}=$

63 0.2) at ambient pressure. Recently, $\sim 2073 \mathrm{~K}$ was achieved at $\sim 10 \mathrm{GPa}$ in a multi-anvil

64 apparatus with (amorphous-)SiC/TiC composites [12]. However, none of the trials could

65 provide a composite suitable to be machined in a tubular shape, which is the most reliable

66 shape for heater in a multi-anvil high pressure cell. A commercial $\mathrm{TiC}-\mathrm{Al}_{2} \mathrm{O}_{3}$ composite

67 (NPA-2, Nippon Tungsten Co.,Ltd) has been applied for in-situ high pressure studies and a

68 temperature of $2250 \mathrm{~K}$ was generated [13]. However, the hardness of NPA-2 makes the

69 fabrication difficult, and thus the cost of a fabricated tubular heater very high.

70 In the present study, we report the protocol we used to synthesize TiC-MgO

71 composite, which was proved to be easy to machine in tubular heaters used to generate high 
72 temperature under high pressure in a multi-anvil apparatus. The TiC-MgO heaters exhibited

73 not only stable ultra-high temperature generation, but also a good X-ray transparency, as

74 tested during in situ radiography and diffraction measurements of liquid Fe-S alloys

75 performed at synchrotron SOLEIL.

\section{2. Experiments}

\section{7}

\section{A. Synthesis of TiC-MgO blocks}

TiC-MgO starting material was prepared by mixing of $\mathrm{TiC}$ and $\mathrm{MgO}$ powders with different TiC concentrations, i.e. 50 and $70 \mathrm{wt} . \%$ (hereafter, we refer it as TiC-50 and TiC70 , respectively), in an agate mortar with either ethanol or acetone. We substituted $15 \mathrm{wt} . \%$ of $\mathrm{MgO}$ by $\mathrm{Mg}(\mathrm{OH})_{2}$ to enhance quality of sintering. The obtained $\mathrm{TiC}-\mathrm{MgO}-\mathrm{Mg}(\mathrm{OH})_{2}$ mixtures were dried at $80^{\circ} \mathrm{C}$ in vacuum to remove remnant acetone/ethanol before molding. The mixtures were compressed at $200 \mathrm{MPa}$ for 5 minutes in a cold isostatic press (CIP) and then calcinated in a gas flow furnace under reducing atmosphere of $\mathrm{Ar}+10 \% \mathrm{H}_{2}$ at $1273 \mathrm{~K}$ for 2-5 hours with a rising rate of $2 \mathrm{~K} / \mathrm{min}$. Fig.1a shows rods recovered after calcination process.

The TiC-MgO blocks were machined by lathe or numerically controlled (NC) milling machine into tube shaped heaters of desired dimensions (Figure 1b). Through the machining processes, it was observed that the machinability improves with increasing $\mathrm{MgO}$ content in the blocks. Further thinning is mechanically possible, while not necessary for our purpose of practical heaters for stable temperature generation in high pressure experiment.

2 Microstructure of recovered TiC-MgO blocks observed by a field emission scanning electron microscope (SEM-FEG) (Zeiss Ultra55) at IMPMC, Sorbonne University, France, 
94 is illustrated in Fig.2. Sintered TiC-MgO blocks consist of TiC grains with $\sim 1-4 \mu \mathrm{m}$ size and

95 finer grains of $\mathrm{MgO} . \mathrm{MgO}$ grains distribute homogeneously in the TiC matrix, while some

96 small clusters can be seen both in $\mathrm{TiC}-70$ and TiC-50. X-Ray powder diffraction

97 measurements were carried out at the X-ray diffraction platform of the IMPMC on a Rigaku

98 MM007HF diffractometer for phase identification. Diffraction patterns of the TiC-MgO

99 show no obvious brucite $\left(\mathrm{Mg}(\mathrm{OH})_{2}\right)$ peaks (Fig. 3), which indicates a complete dehydration

100 during annealing. No peaks of $\mathrm{TiO}$ (partial reduction of $\mathrm{MgO}$ by $\mathrm{TiC}$ ) [14], $\mathrm{TiO}_{2}$ (oxidization

101 of $\mathrm{TiC}$ ), $\mathrm{MgTi}_{2} \mathrm{O}_{5}, \mathrm{MgTiO}_{3}$ and $\mathrm{Mg}_{2} \mathrm{TiO}_{4}$ resulting from $\mathrm{TiO}_{2}-\mathrm{MgO}$ reaction [15] were

102 observed in the diffraction pattern. A small amount of $\mathrm{Ti}$ and $\mathrm{TiC}_{0.5} \mathrm{H}_{0.707}$ might be present

103 in the bulk material (Fig. 3), as results of the reducing sintering atmosphere provided by

$104 \mathrm{Ar}+10 \% \mathrm{H}_{2}[16,17]$.

\section{B. Tests of heating performances}

106 We tested heating performance of the sintered $\mathrm{TiC}-\mathrm{MgO}$ heater by carrying out high

107 pressure and high temperature experiments in the Kawai-type multi-anvil apparatus installed

108 at Institute for Planetary Materials, Okayama University, Japan. Fig. 4a and Fig. 4b show

109 schematic view of the cell assemblies. Pressure of $10 \mathrm{GPa}$ was generated using WC cubes

110 with a $4 \mathrm{~mm}$ truncated edge length. Table 1 summarizes experimental specifications

111 including one run for which $\mathrm{TiC}-\mathrm{Al}_{2} \mathrm{O}_{3}(50 \mathrm{wt} . \%)$ composite heater (obtained following the

112 same sintering procedure with TiC-MgO composite) was used as a possible alternative for

113 TiC-MgO composite. 


\section{Application to in situ study on liquid Fe alloys}

115 In situ radiography and diffraction experiments were conducted in the DIA-type multi116 anvil apparatus installed at beamline PSICHE, SOLEIL, France. Fig. 4c shows the cell

117 assembly used in these experiments. Boron-15 wt.\% MgO composite (Xie et al., under review)

118 was used both for pressure medium and gaskets in order to minimize the X-ray absorption of

119 the sample environment. Pressure was generated by WC cubes with a $4 \mathrm{~mm}$ truncated edge

120 length and determined through the equation of state of $\mathrm{MgO}$ marker placed at the

121 thermocouple junction. The Fe-FeS powder mixture starting material was loaded in a 122 sapphire capsule with caps made of BN.

123 After having reached the target load, heating was performed with voltage control. X-ray

124 shadowgraphs of cell assembly were collected on a fluorescence screen and detected by a

125 high-speed CCD camera (C9300, Hamamatsu Co., Japan). Energy dispersive diffraction

126 patterns were collected on a germanium solid-state detector for increasing temperature

127 (typical temperature step size $100 \mathrm{~K}$ ). Upon complete melting, assessed by appearance of a 128 diffuse scattering signal and disappearance of diffraction peak, energy-dispersive diffraction 129 patterns were collected in a $2 \theta$ angle range from 2.5 to $25^{\circ}$, with a step size of $0.2^{\circ}$ by using 130 a germanium solid-state detector. Such combined angle- and energy-dispersive structural 131 analysis and refinement (CAESAR) method allows recording the diffuse scattering signal 132 from the liquid sample over a large reciprocal space $(Q)$ range [18]. 


\section{3. Result and discussion}

\section{A. Results of the heating performance tests}

135 Fig. 5a shows the power-temperature relationships recorded during the test heating runs

136 employing $\mathrm{TiC}-\mathrm{Al}_{2} \mathrm{O}_{3}$ and $\mathrm{TiC}-\mathrm{MgO}$ heaters, while Fig. 5b shows their Arrhenius plots of

137 electrical conductivity. Run with $\mathrm{TiC}-\mathrm{Al}_{2} \mathrm{O}_{3}$ composite heater was well performed up to 1970

$138 \mathrm{~K}$, temperature above which the thermocouple started having an anomalous reading. We

139 ascribed this malfunctioning to the melting of the heater, as confirmed by inspection of the

140 recovered cell (Fig. 6a). As this melting temperature is lower than the temperature achieved

141 using NPA-2 in [13], the observed melting might have been caused by minor impurities

142 associated to the sintering process. As for runs with $\mathrm{TiC}-\mathrm{MgO}$ composites, temperature is

143 very stable $( \pm 2 \mathrm{~K})$ till the highest temperature reached in each run. No obvious melting was

144 observed in the images of the recovered cells (Fig. 6b, c, d). The higher heating efficiency of

145 runs $1 \mathrm{~K} 2881$ and $1 \mathrm{~K} 2890$ is a direct consequence of the lower thermal conductivity of the

146 boron-15 wt.\% $\mathrm{MgO}$ pressure medium used in these two runs (Fig. 2a), with respect to

147 conventional $\mathrm{MgO}-5$ wt. $\% \mathrm{Cr}_{2} \mathrm{O}_{3}$ pressure medium used in the other runs (Xie et al., under

148 review).

149 In both test runs of 1K2892 and 1K2894, for which we used the same cell assembly (Fig.

150 4b), we successfully generated high temperatures above $2100 \mathrm{~K}$. Obviously, TiC-70 has

151 higher electrical conductivity than TiC-50 due to the higher content of conductive TiC.

152 During heating, the electrical conductivity of the TiC-MgO heaters increased until $~ 700 \mathrm{~K}$

153 mainly as an outcome of the compaction process (i.e. improvement of contact between 154 heating elements and electrodes by deformation of materials). Then, we observed the 
155 opposite trend, with conductivity decreasing with increasing temperature, which we attribute 156 to the metal-like electric conduction of TiC. On the contrary, the $\mathrm{TiC}-\mathrm{Al}_{2} \mathrm{O}_{3}$ heater $(1 \mathrm{~K} 2881)$

157 showed continuous increase of electrical conductivity, possibly due to the high strength of 158 corundum and limited plastic deformation under high temperature. Both heating of 1 K2892 159 and 1K2894 was intentionally terminated when we started observing an increase of heater 160 resistance accompanied by a decrease of temperature (Fig.5a). No temperature instability was

161 recorded until then. No obvious signature of heater melting was found in the SEM image of 162 recovered assembly (Fig.6b, c, d). Since our heater may contain a small amount of Ti-C-H 163 phases, whose melting temperature, close to that of metallic $\mathrm{Ti}(\sim 2000 \mathrm{~K})$, is much lower 164 than that of $\mathrm{TiC}$, we could ascribe the failure of heater to the melting of these minor phases. 165 In summary, both $\mathrm{TiC}-70$ and $\mathrm{TiC}-50$ were proved to be suitable heaters, capable of 166 generating temperatures exceeding $2000 \mathrm{~K}$ in multi-anvil apparatus. Given the similar 167 heating performance, TiC-50 heater is more recommended because of its higher 168 machinability. Furthermore, its lower electric conductivity allows making thicker tubular 169 heaters, enhancing mechanical strength of heating elements.

170 As the resistivity of $\mathrm{TiC}-\mathrm{MgO}$ heaters is comparable to that of graphite heaters, 171 conventional power supply system can be used, without requiring any modification.

\section{B. Results of applications to in situ study on liquid Fe alloys}

173 The X-ray transparency of $\mathrm{TiC}-\mathrm{MgO}$ heaters was compared with that of classic graphite

174 heaters by using the same cell assembly. It is well known that graphite has the highest X-ray 175 transparency among the available heaters used for experimentation in large volume press, 176 due to the small atomic number of carbon. As shown in Fig. 7, radiographies of Fe-S sample 
177 surrounded by TiC-MgO heater are as clear as those obtained using graphite heater. Sharp

178 contrast among materials in the cell is very beneficial for quick identification of sample 179 position and geometry.

180 Thanks to the use of TiC-MgO heaters we collected good quality CAESAR scans, with

181 high signal to noise ratio, over a pressure and temperature range inaccessible with graphite 182 heaters (Table 1 and Fig. 8). Data obtained using TiC-MgO heaters and using graphite heaters

183 are overall comparable, with intensity in the case of $\mathrm{TiC}-\mathrm{MgO}$ only slightly lower than for 184 graphite heater. The high quality diffraction data over a large energy and angular range enable 185 to obtain accurate structure factor over a large range of $Q$. Concerning stability and endurance 186 as a furnace, the TiC-MgO composite heater stably operated at $10 \mathrm{GPa}$ for more than 9 hours, 187 at temperatures ranging from 1215 to $2145 \mathrm{~K}$, with stable temperature maintained for about 1881 hour for each temperature step (100 K increment) (MA41), granting enough time for 189 thorough investigation and data collections.

\section{Acknowledgment}

191 We would like to thank B. Baptiste for his help with XRD measurements. The 192 synchrotron radiation experiments were performed under SOLEIL proposals 20170506, 19320171307 and 20181797. This project has received funding from the European Research 194 Council (ERC) under the European Union's Horizon 2020 research and innovation program 195 (Grant agreement No. 724690). 


\section{Reference}

198 [1] Ito, E., 2007. 2.08 Theory and practice-multianvil cells and high-pressure experimental 199 method. Mineral physics. In: Treatise on Geophysics, vol. 2, pp. 197-230.

200 [2] Katsura, T., 2007. Phase-relation studies of mantle minerals by in situ X-ray diffraction 201 using multianvil apparatus. In: Advances in High-Pressure Mineralogy: Special Papers202 geological society of America, 421, pp.189.

203 [3] Kanzaki M., Kurita K., Fujii T., Kato T., Shimomura O., Akimoto S., 1987. A new 204 technique to measure the viscosity and density of silicate melts at high pressure. In: 205 Geophysics Monograph 39: High-Pressure Research in Mineral Physics, pp. 195-200.

206 [4] Xie, L., Yoneda, A., Yamazaki, D., Manthilake, G., Higo, Y., Tange, Y., Guignot, N., 207 King, A., Scheel, M., Andrault, D., 2020. Formation of bridgmanite-enriched layer at the 208 top lower-mantle during magma ocean solidification. Nature Comm. 11(1), pp.1-10.

209 [5] Sanloup, C., Guyot, F., Gillet, P., Fiquet, G., Mezouar, M., Martinez, I., 2000. Density 210 measurements of liquid Fe-S alloys at high-pressure. Geophys. Res. Lett., 27, pp.811-814.

211 [6] Sakamaki, T., Ohtani, E., Urakawa, S., Suzuki, A., Katayama, Y., 2009. Measurement of 212 hydrous peridotite magma density at high pressure using the X-ray absorption method. 213 Earth Planet. Sci. Lett., 287, pp.293-297.

214 [7] Xie, L., Yoneda, A., Yoshino, T., Yamazaki, D., Tsujino, N., Higo, Y., Tange, Y., Irifune, 215 T., Shimei, T., Ito, E., 2017. Synthesis of boron-doped diamond and its application as a 216 heating material in a multi-anvil high-pressure apparatus. Rev. Sci. Inst., 88, p.093904.

217 [8] Chang, R., Graham, L.J., 1966. Low-temperature elastic properties of ZrC and TiC. J. $218 \quad$ Appl. Phys., 37, pp.3778-3783. 
219 [9] Sokolov, P. S., Mukhanov, V. A., Chauveau, T., Solozhenko, V. L., 2012. On melting of

220 silicon carbide under pressure. J. Superhard Materials, 34(5), pp.339-341.

221 [10] Yoshida M, Onodera A., 1993. Pressure-induced phase transition in SiC. Phys. Rev. B 222 Condens. Matter., 48, pp.10587-10590.

223 [11] Taniguchi, T., Akaishi, M., Kanke, Y., Yamaoka, S., 2004. TiC-diamond composite 224 disk-heater cell assembly to generate temperature of $2000^{\circ} \mathrm{C}$ in a large-volume belt-type 225 high-pressure apparatus at $10 \mathrm{GPa}$. Rev. Sci. Inst., 75(6), pp.1959-1962.

226 [12] Guan, L., Schwarz, M., Zhang, R., Kroke, E., 2016. Polymer-precursor-derived (am-) $227 \mathrm{SiC} / \mathrm{TiC}$ composites for resistive heaters in large volume multi-anvil high pressure/high228 temperature apparatus. High Press. Res., 36(2), pp.167-186.

229 [13] Terasaki, H., Rivoldini, A., Shimoyama, Y., Nishida, K., Urakawa, S., Maki, M., 230 Kurokawa, F., Takubo, Y., Shibazaki, Y., Sakamaki, T., Machida, A., 2019. Pressure and 231 composition effects on sound velocity and density of core-forming liquids: implication 232 to core compositions of terrestrial planets. J. Geophys. Res.: Planets, 124(8), pp.22722332293.

234 [14] Ueltz, H.F.G., 1950. Sintering reactions in MgO-TiC mixtures. J. Amer. Ceram. Soc., 23533, pp.340-344.

236 [15] Wechsler, B.A., Navrotsky, A., 1984. Thermodynamics and structural chemistry of 237 compounds in the system $\mathrm{MgO}-\mathrm{TiO}_{2}$. J. Solid State Chem., 55(2), pp.165-180.

238 [16] Fedorov, L.Y., Karpov, I.V., Ushakov, A.V., Lepeshev, A.A., 2015. Influence of 239 pressure and hydrocarbons on carbide formation in the plasma synthesis of TiC 240 nanoparticles. Inorganic Materials, 51(1), pp.25-28. 
241 [17] Renaudin, G., Yvon, K., Dolukhanyan, S.K., Aghajanyan, N.N., Shekhtman, V.S., 2003.

242 Crystal structures and thermal properties of titanium carbo-deuterides as prepared by

243 combustion synthesis. J. Alloys Compounds, 356, pp.120-127.

244 [18] Wang, Y., Uchida, T., Von Dreele, R., Rivers, M.L., Nishiyama, N., Funakoshi, K.I.,

245 Nozawa, A., Kaneko, H., 2004. A new technique for angle-dispersive powder diffraction

246 using an energy-dispersive setup and synchrotron radiation. J. App. Crystal., 37, pp.947-

247956. 
Figure captions

249 Fig.1 Pictures of synthesized TiC-MgO blocks (a) and machined tubes (b). The color 250 difference in (b) is due to the low/high $\mathrm{TiC}$ (50/70 wt.\%) concentration. We successfully 251 obtained tube shape heaters of 3.5-5.0 mm length with OD/ID of 2.0-2.4/1.6 mm and 2.4/1.8 252 (OD: outer diameter, ID: inner diameter).

254 Fig.2 Electron back scattered images of sintered rods of TiC-70 (a, b) and TiC-50 (c, d). 255 Bright and dark grains correspond to $\mathrm{TiC}$ and $\mathrm{MgO}$, respectively.

Fig. 3 X-ray diffraction patterns of the sintered TiC-MgO. Monochromatic light from a 258 rotating Mo anode $(\lambda \mathrm{K} \alpha 1=0.709319 \AA)$ was used. The systematically observed second 259 peaks marked by vertical sticks are diffraction peaks from $\mathrm{K} \alpha 2$ emission $(\lambda \mathrm{K} \alpha 2=0.713609$

$260 \AA$ A). No obvious shift of TiC or MgO peaks was observed, suggesting no chemical reaction or 261 inter-grain diffusion. The black arrows marked the indexed peaks of $\mathrm{TiC}_{0.5} \mathrm{H}_{0.707}$, by 262 assuming same peak positions between $\mathrm{TiC}_{0.5} \mathrm{H}_{0.707}$ and $\mathrm{TiC}_{0.5} \mathrm{D}_{0.707}$.

264 Fig. 4 Cell assemblies used for high pressure heater test experiments with boron-MgO 265 (boron-15 wt.\% $\mathrm{MgO}$ ) (a, c) and $\mathrm{MgO}-\mathrm{Cr}_{2} \mathrm{O}_{3}\left(\mathrm{MgO}-5\right.$ wt.\% $\left.\mathrm{Cr}_{2} \mathrm{O}_{3}\right)$ (b) as pressure medium. 266 Please note that heaters with different dimensions were used in cell assembly (a) (OD/ID: 267 2.0/1.6 mm) and cell assemblies (b) and (c) (OD/ID: 2.4/1.6 mm). Temperature was 268 monitored with a $\mathrm{W}_{97} \mathrm{Re}_{3}-\mathrm{W}_{75} \mathrm{Re}_{25}$ thermocouple (TC) whose junction was indicated by 269 block dots. 
271 Fig. 5 The power-temperature diagrams (a) and Arrhenius plots of electrical conductivity (b) 272 of TiC-MgO/Al ${ }_{2} \mathrm{O}_{3}$ heaters. Also plotted in (a) is the heater resistance measured for runs $2731 \mathrm{~K} 1892$ and $1 \mathrm{~K} 2894$. Noted that the resistance of $1 \mathrm{~K} 2892$ is rescaled for $0.01 \Omega$ for 274 convenience. Experimental specifications are summarized in Table 1. Electrical conductivity 275 was calculated from the measured total resistance by assuming electrode resistance and 276 changes in heater dimensions are negligible during compression. The kinks as illustrated by 277 arrows represent keeping of a constant temperature during near uniform heating. The circled 278 data in light gray for run $1 \mathrm{~K} 2881$ corresponds to an anomalous temperature reading 279 associated to the melting of the heater.

281 Fig 6. Electron back scattered image of recovered samples of run 1K2881 (a),1K2890 (b), $2821 \mathrm{~K} 2894$ (c) and magnified image of rectangle area in 1K2894 (d). The same cell assembly

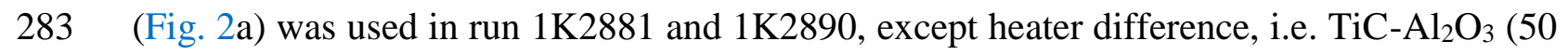
284 wt.\%) heater in run 1K2881 and TiC-50 heater in run 1K2890. TiC-70 heater was used in $2851 \mathrm{~K} 2894$ with cell assembly shown in Fig. 2b.

287 Fig. 7 Images of cell assemblies obtained by X-ray radiography. Cell assembly with TiC-50 288 heater (MA41) before compression (a) and at $10.8 \mathrm{GPa}$ (c) in comparison with the same cell 289 assembly but with graphite heater, before compression (b) and at 7.4 GPa (d). 
291 Fig. 8 Normalized CAESAR scans of liquid Fe-S alloys collected at $10.0 \mathrm{GPa}$ and $1400 \mathrm{~K}$ 292 using the TiC-50 heater (a) (MA41), 6.4 GPa and $1400 \mathrm{~K}$ using a graphite heater (b) and the 293 averaged intensity of all two theta (c). Note that the higher pressure of the experiment using 294 TiC-50 heater contributes partially to the observed lower diffuse scattering intensity 295 compared with the experiment using graphite heater. 
296 Table 1: Summary of maximal temperature generated at high pressure using the TiC heater.

297 The prefixes " $1 \mathrm{k}$ " and "MA" in the run numbers correspond to the 1000-ton press installed

298 at the Institute for Planetary Materials (Okayama University), and multi-anvil press installed

299 at the SOLEIL synchrotron facility, respectively. TiC- $\mathrm{Al}_{2} \mathrm{O}_{3}, \mathrm{TiC}-50$ and $\mathrm{TiC}-70$ refers to

300 heaters made of TiC mixed with 50 wt. $\% \mathrm{Al}_{2} \mathrm{O}_{3}, 50$ wt. $\%$ and 30 wt.\% $\mathrm{MgO}$, respectively.

301 Cell a, b and c refer to assembly showed in Fig. 4. The heater size is defined by outer diameter,

302 inner diameter, and length (in this order).

\begin{tabular}{|c|c|c|c|c|c|}
\hline Run NO & Heater material & Cell & Heater size $(\mathrm{mm})$ & $\mathrm{P}(\mathrm{GPa})$ & Highest T (K) \\
\hline \multicolumn{6}{|c|}{ Heater test experiments } \\
\hline $1 \mathrm{~K} 2881$ & TiC- $\mathrm{Al}_{2} \mathrm{O}_{3}$ & $\mathrm{a}$ & \multirow{2}{*}{ 2.0/1.6/4.0 } & \multirow{4}{*}{$\sim 10$} & 1970 \\
\hline $1 \mathrm{~K} 2890$ & TiC- 50 & $\mathrm{a}$ & & & 1770 \\
\hline $1 \mathrm{~K} 2892$ & TiC- 50 & $\mathrm{~b}$ & \multirow{2}{*}{ 2.4/1.6/4.0 } & & 2170 \\
\hline $1 \mathrm{~K} 2894$ & TiC- 70 & $\mathrm{~b}$ & & & 2250 \\
\hline \multicolumn{6}{|c|}{ In-situ X-ray experiments } \\
\hline MA41 & TiC- 50 & $\mathrm{c}$ & 2.2/1.6/3.7 & 10 & 2145 \\
\hline MA45 & TiC- 70 & $\mathrm{c}$ & $2.2 / 1.6 / 3.7$ & 11.5 & 1900 \\
\hline MA55 & TiC- 50 & $\mathrm{c}$ & $2.4 / 1.8 / 3.5$ & 14 & 2070 \\
\hline
\end{tabular}


(a)

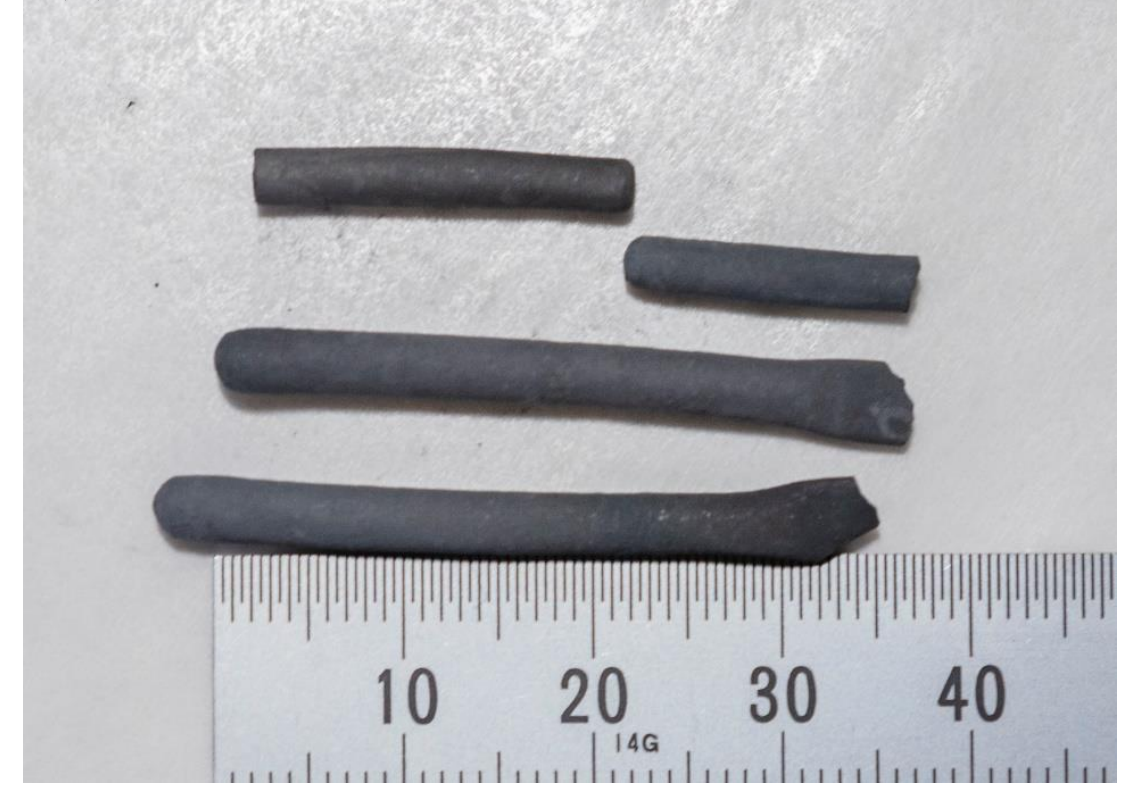

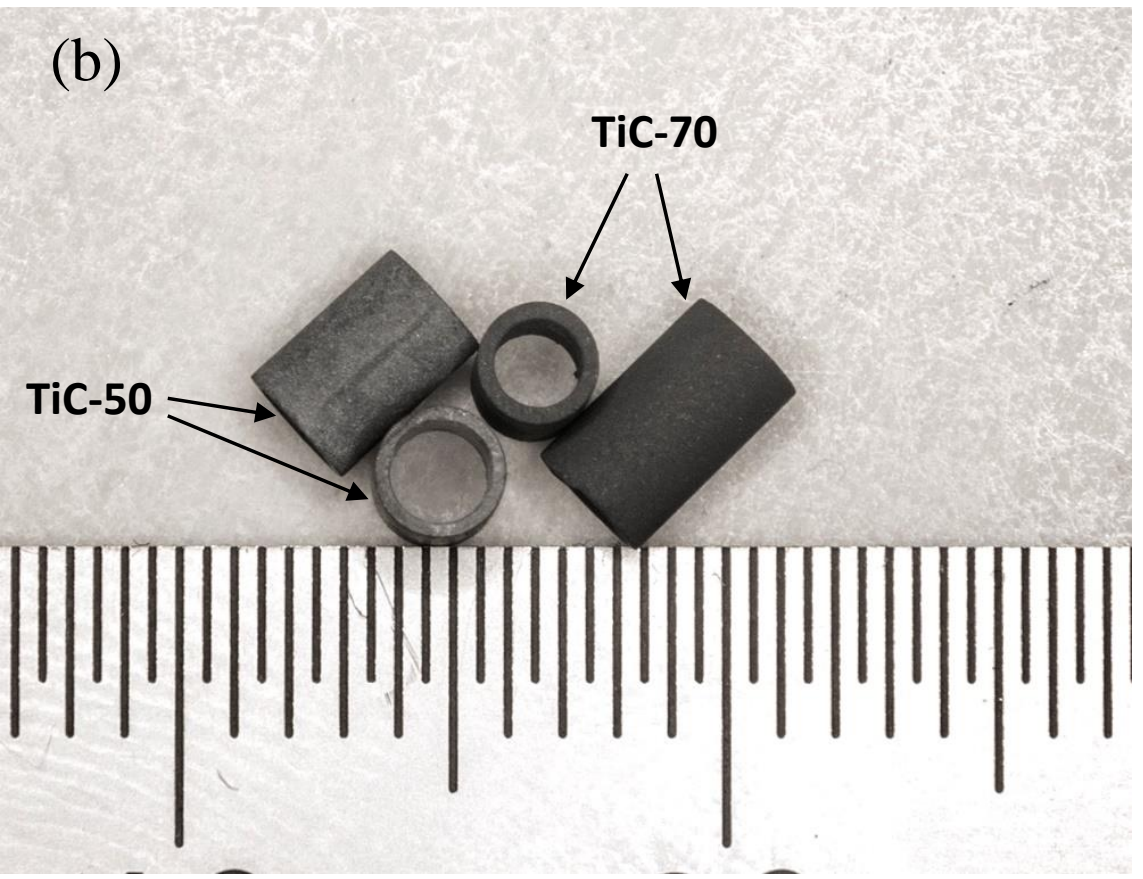

Fig.1 Pictures of synthesized TiC-MgO blocks (a) and machined tubes (b). The color difference in (b) is due to the low/high TiC (50/70 wt.\%) concentration. We successfully obtained tube shape heaters of 3.5-5.0 mm length with OD/ID of 2.02.4/1.6 $\mathrm{mm}$ and 2.4/1.8 (OD: outer diameter, ID: inner diameter). 

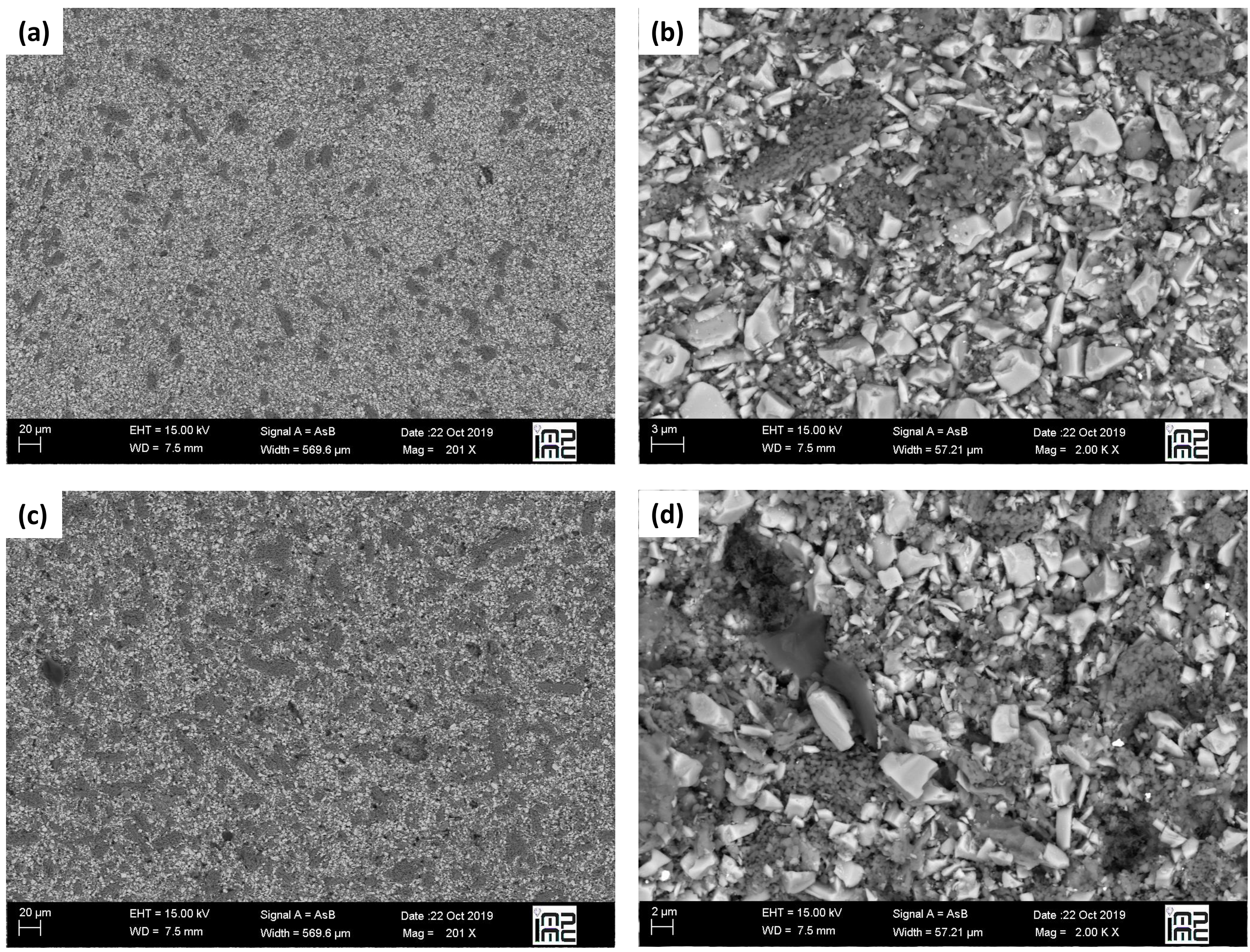

Fig.2 Electron back scattered images of sintered rods of TiC-70 (a, b) and TiC-50 (c, d). Bright and dark grains correspond to TiC and MgO, respectively. 


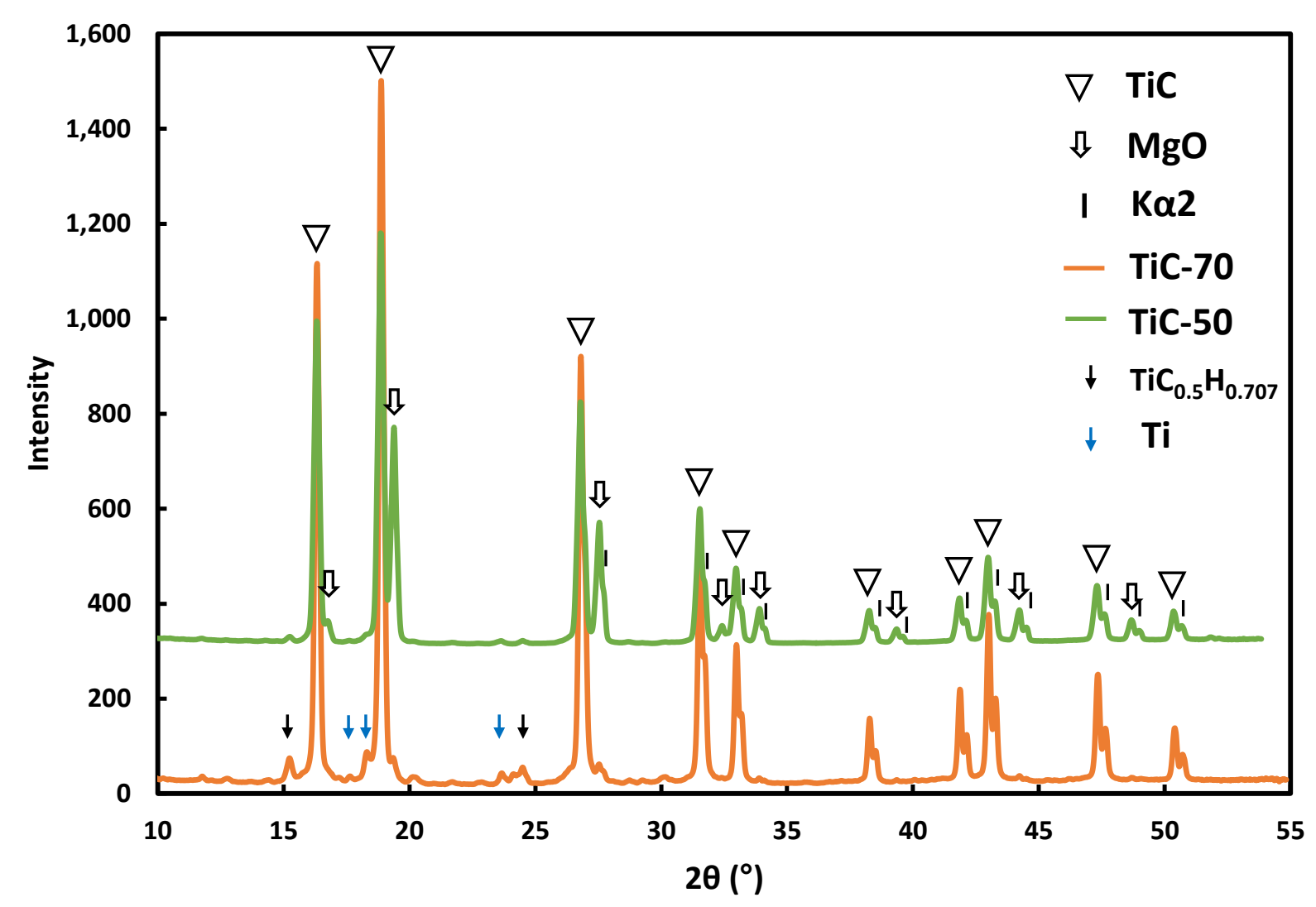

Fig. $3 \mathrm{X}$-ray diffraction patterns of the sintered TiC-MgO. Monochromatic light from a rotating Mo anode $(\lambda K \alpha 1=0.709319 \AA)$ was used. The systematically observed second peaks marked by vertical sticks are diffraction peaks from $K \alpha 2$ emission $(\lambda K \alpha 2=0.713609$ $\AA ̊$ ). No obvious shift of TiC or MgO peaks was observed, suggesting no chemical reaction or inter-grain diffusion. The black arrows marked the indexed peaks of $\mathrm{TiC}_{0.5} \mathrm{H}_{0.707}$, by assuming same peak positions between $\mathrm{TiC}_{0.5} \mathrm{H}_{0.707}$ and $\mathrm{TiC}_{0.5} \mathrm{D}_{0.707}$. 
(a)

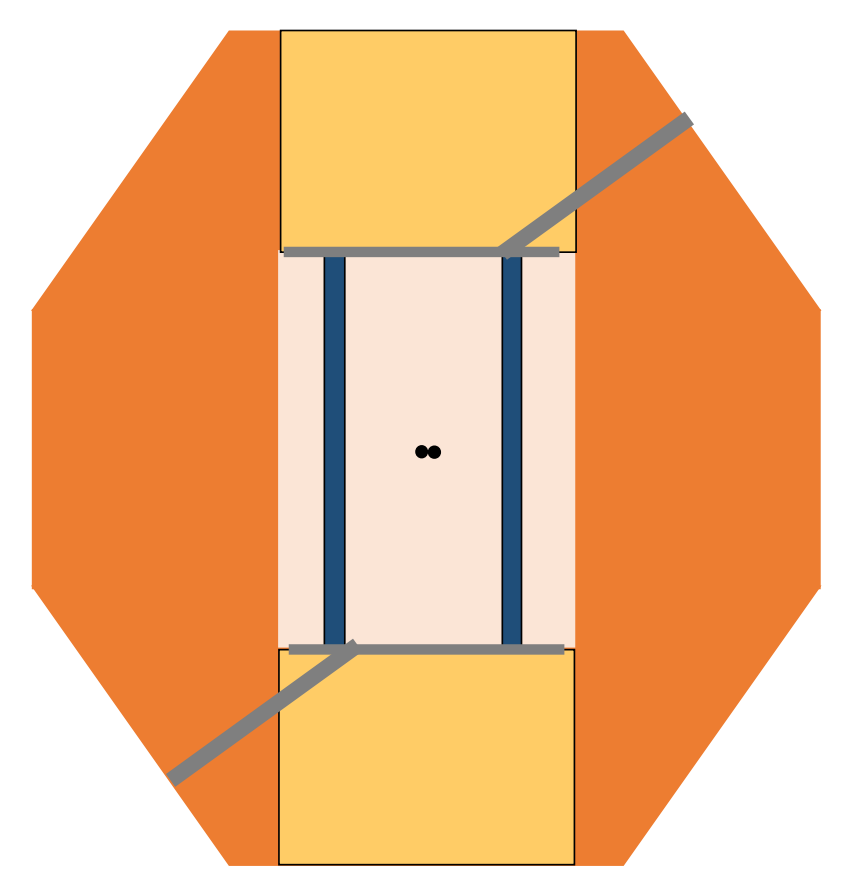

(c)

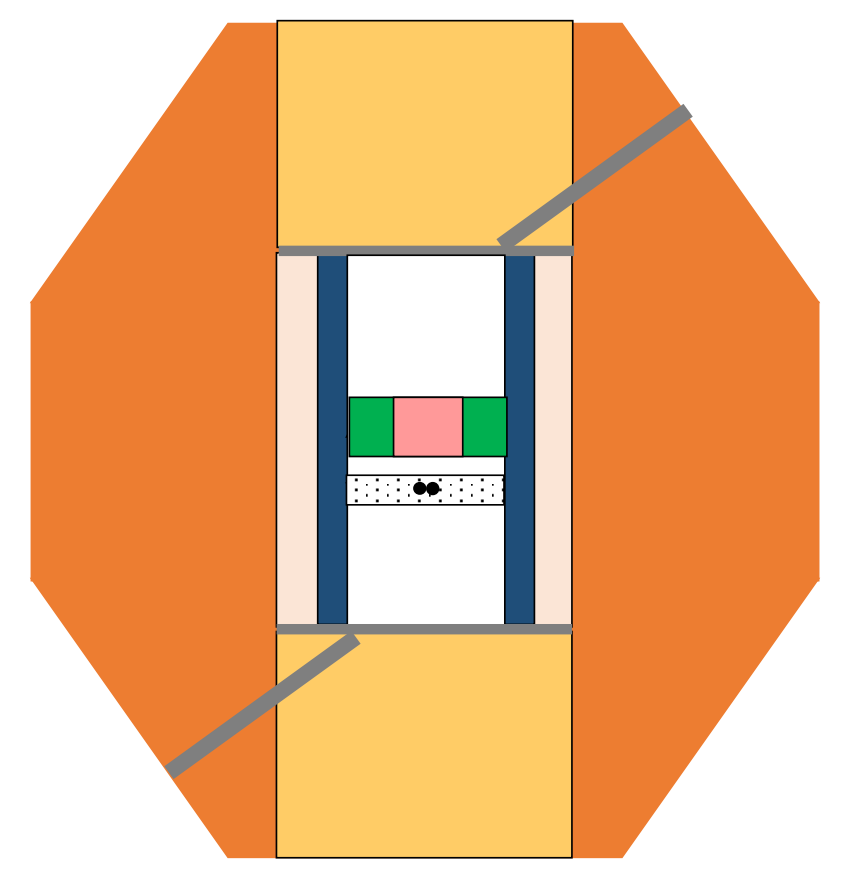

(b)

$2.0 \mathrm{~mm}$

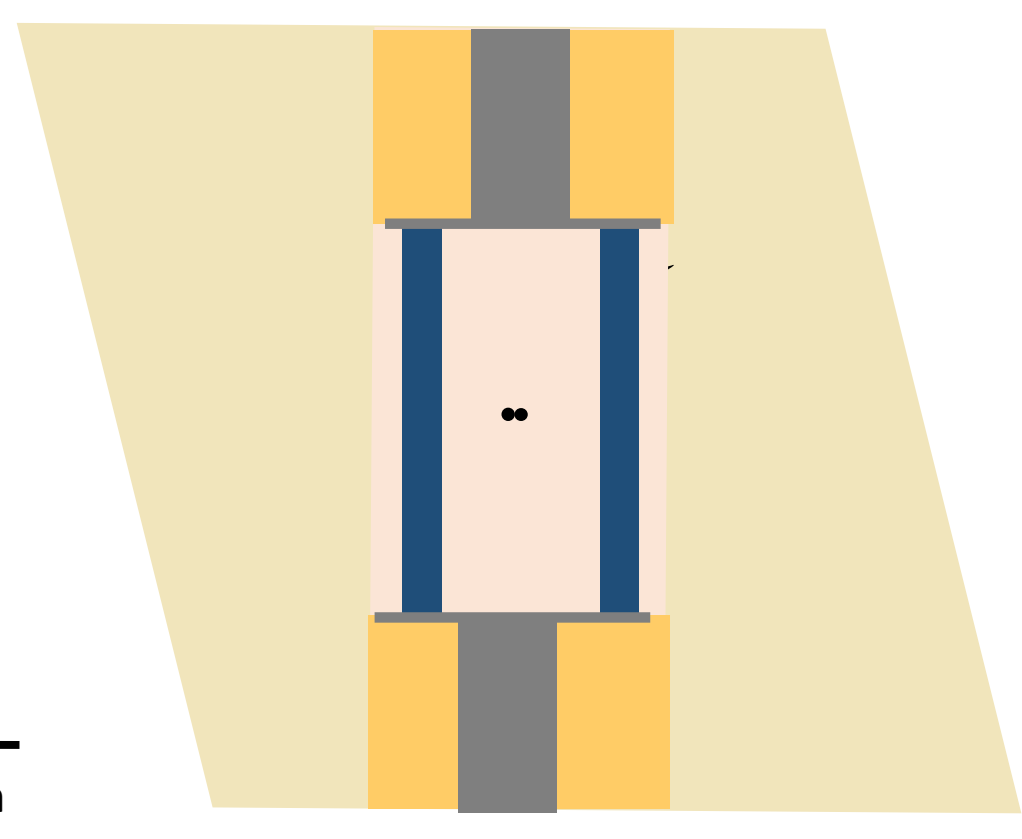

Fig. 4 Cell assemblies used for high pressure heater test experiments with boron-MgO (boron15 wt.\% MgO) (a, c) and $\mathrm{MgO}-\mathrm{Cr}_{2} \mathrm{O}_{3}\left(\mathrm{MgO}-5 \mathrm{wt} . \% \mathrm{Cr}_{2} \mathrm{O}_{3}\right)(\mathrm{b})$ as pressure medium. Please note that heaters with different dimensions were used in cell assembly (a) (OD/ID: 2.0/1.6 mm) and cell assemblies (b) and (c) (OD/ID: 2.4/1.6 mm). Temperature was monitored with a $\mathrm{W}_{97} \mathrm{Re}_{3}$ $\mathrm{W}_{75} \mathrm{Re}_{25}$ thermocouple (TC) whose junction was indicated by block dots. 



Fig. 5 The power-temperature diagrams (a) and Arrhenius plots of electrical conductivity (b) of $\mathrm{TiC}-\mathrm{MgO} / \mathrm{Al}_{2} \mathrm{O}_{3}$ heaters. Also plotted in (a) is the heater resistance measured for runs $1 \mathrm{~K} 1892$ and $1 \mathrm{~K} 2894$. Noted that the resistance of $1 \mathrm{~K} 2892$ is rescaled by $0.01 \Omega$ for convenience. Experimental specifications are summarized in Table 1. Electrical conductivity was calculated from the measured total resistance by assuming electrode resistance and changes in heater dimensions are negligible during compression. The kinks as illustrated by arrows represent keeping of a constant temperature during near uniform heating. The circled data in light gray for run $1 \mathrm{~K} 2881$ corresponds to an anomalous temperature reading associated to the melting of the heater. 



Fig 6. Electron back scattered image of recovered samples of run 1K2881 (a),1K2890 (b), $1 \mathrm{~K} 2894$ (c) and magnified image of rectangle area in $1 \mathrm{~K} 2894$ (d). The same cell assembly (Fig. 2a) was used in run $1 \mathrm{~K} 2881$ and $1 \mathrm{~K} 2890$, except heater difference, i.e. $\mathrm{TiC}_{-} \mathrm{Al}_{2} \mathrm{O}_{3}$ (50 wt.\%) heater in run $1 \mathrm{~K} 2881$ and TiC-50 heater in run $1 \mathrm{~K} 2890$. TiC-70 heater was used in $1 \mathrm{~K} 2894$ with cell assembly shown in Fig. 2 b. 

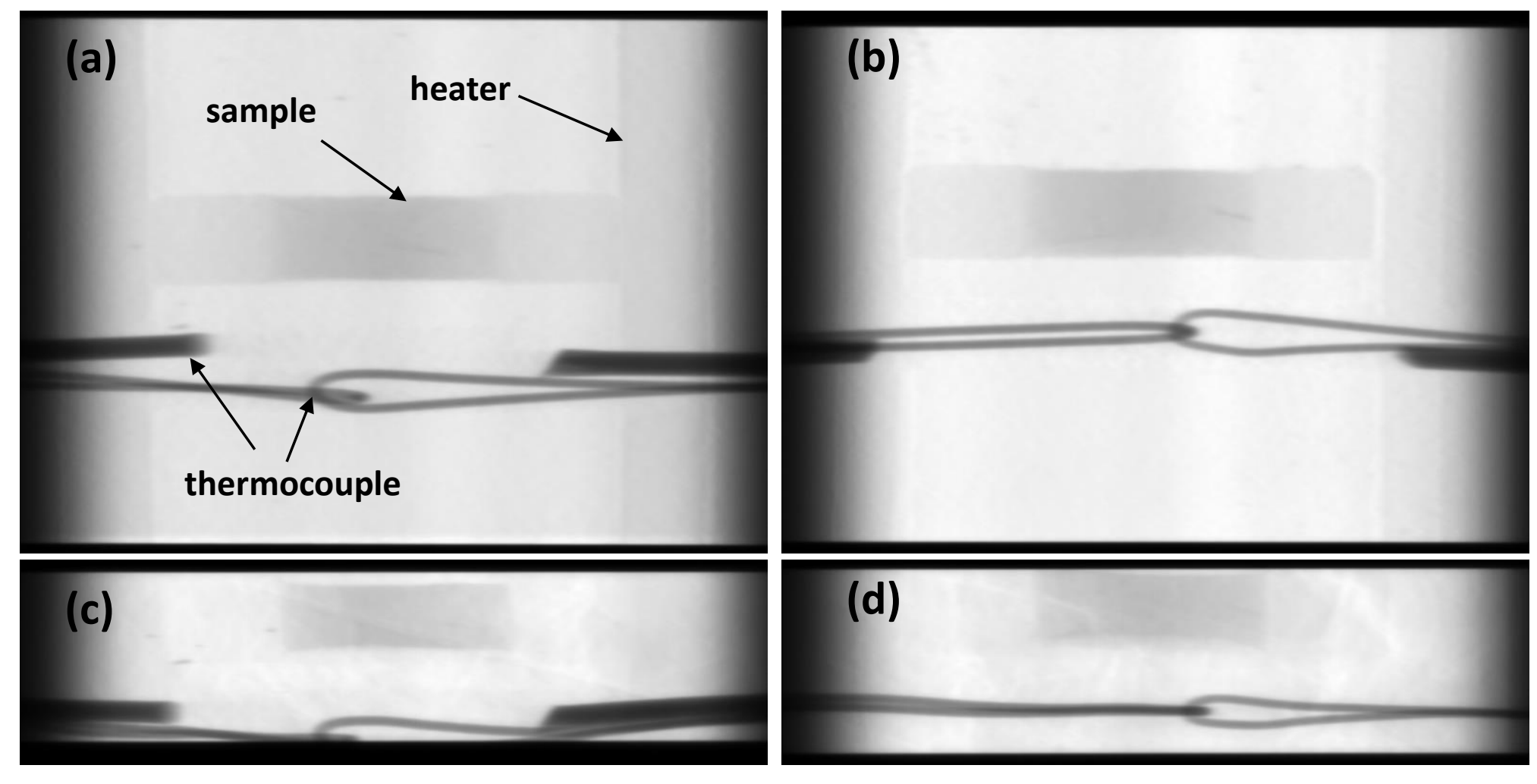

Fig. 7 Images of cell assemblies obtained by X-ray radiography. Cell assembly with TiC-50 heater (MA41) before compression (a) and at $10.8 \mathrm{GPa}$ (c) in comparison with the same cell assembly but with graphite heater, before compression (b) and at 7.4 GPa (d). 




Fig. 8 Normalized CAESAR scans of liquid Fe-S alloys collected at $10.0 \mathrm{GPa}$ and $1400 \mathrm{~K}$ using the TiC-50 heater (a) (MA41), 6.4 GPa and $1400 \mathrm{~K}$ using a graphite heater (b) and the averaged intensity of all two theta (c). Note that the higher pressure of the experiment using TiC-50 heater contributes partially to the observed lower diffuse scattering intensity compared with the experiment using graphite heater. 